\title{
Electron cross talk and asymmetric electron distributions near the Earth's bowshock
}

\author{
J. J. Mitchell ${ }^{1}$, S. J. Schwartz ${ }^{1}$, and U. Auster ${ }^{2}$ \\ ${ }^{1}$ Space and Atmospheric Physics Group, Blackett Laboratory, Imperial College London, London, SW7 2AZ, UK \\ ${ }^{2}$ Institut für Geophysik and Extraterrestrische Physik, TUBS, Braunschweig, Germany \\ Correspondence to: J. J. Mitchell (j.mitchell@imperial.ac.uk)
}

Received: 10 May 2010 - Revised: 28 November 2011 - Accepted: 2 January 2012 - Published: 6 March 2012

\begin{abstract}
Electron distributions in the magnetosheath display a number of far from equilibrium features. It has been suggested that one factor influencing these distributions may be the large distances separating locations at which electrons with different energies and pitch angles must cross the bowshock in order to reach a given point in the magnetosheath. The overall heating requirements at these distant locations depends strongly on the shock geometry. In the absence of collisions or other isotropization processes this suggests that the convolution of electrons arriving from different locations should give rise to asymmetries in the distribution functions. Moreover, such cross-talk could influence the relative electron to ion heating, rendering the shock heating problem intrinsically non-local in contrast to classic shock physics. Here, we study electron distributions measured simultaneously by the Plasma Electron and Current Experiment (PEACE) on board the Cluster spacecraft and the Electrostatic Analyser (ESA) on board THEMIS b during a time interval in which both the Cluster spacecraft and THEMIS $b$ are in the magnetosheath, close to the bowshock, and during which the local magnetic field orientation makes it likely that electron trajectories may connect both spacecraft. We find that the relevant portions of the velocity distributions of such electrons measured by each spacecraft display remarkable similarities. We map trajectories of electrons arriving at each spacecraft back to the locations at which they crossed the bowshock, as a function of pitch angle and energy. We then use the Rankine-Hugoniot relations to estimate the heating of electrons and compare this with temperature asymmetries actually observed. We conclude that the electron distributions and temperatures in the magnetosheath depend heavily on non-local shock properties.
\end{abstract}

Keywords. Interplanetary physics (Planetary bow shocks)

\section{Introduction}

The supersonic solar wind is interrupted upstream of the Earth at the bowshock, where the plasma's flow is slowed, heated and deflected about the magnetopause, which separates the solar and terrestrial magnetic fields. At the bowshock the change in temperature, bulk velocity, magnetic field, and density agree to some extent with the RankineHugoniot relations (de Hoffmann and Teller, 1950; Kivelson and Russell, 1995). These, however, suffer from a number of inadequacies, including the inability to treat separately electron and ion species, and the assumption of isotropic heating. Furthermore, kinetic effects are ignored, and the adiabatic equation of state is assumed: that is pressure $P$ and density $\rho$ are related by $P \propto \rho^{\gamma}$, where $\gamma=5 / 3$ for a monatomic gas. Numerous attempts have been made to study these shortfalls and provide observational and theoretical insights (Feldman et al., 1983; Kennel et al., 1985; Scudder et al., 1986a,b,c; Lembège et al., 2004).

Properties within the magnetosheath are known to be influenced by conditions upstream at the bowshock (Němeček et al., 2000; Longmore et al., 2005). In this paper we will investigate the impact of the global, curved nature of the bow shock on the shock-related electron heating and dynamics. This builds on suggestions based on observations of magnetosheath electron distributions (Paschmann et al., 1980; Feldman et al., 1983; Masood and Schwartz, 2008) that the electron heating is influenced by intrinsically global processes.

The magnetosheath which lies between the bowshock and the magnetopause exhibits electron velocity distributions that are typically far from Maxwellian. Feldman et al. (1983) showed that these distributions are characterised by flattopped shape and temperature anisotropy. Numerous mechanisms at the bowshock may contribute to electron heating such as acceleration by the cross shock potential (Goodrich 


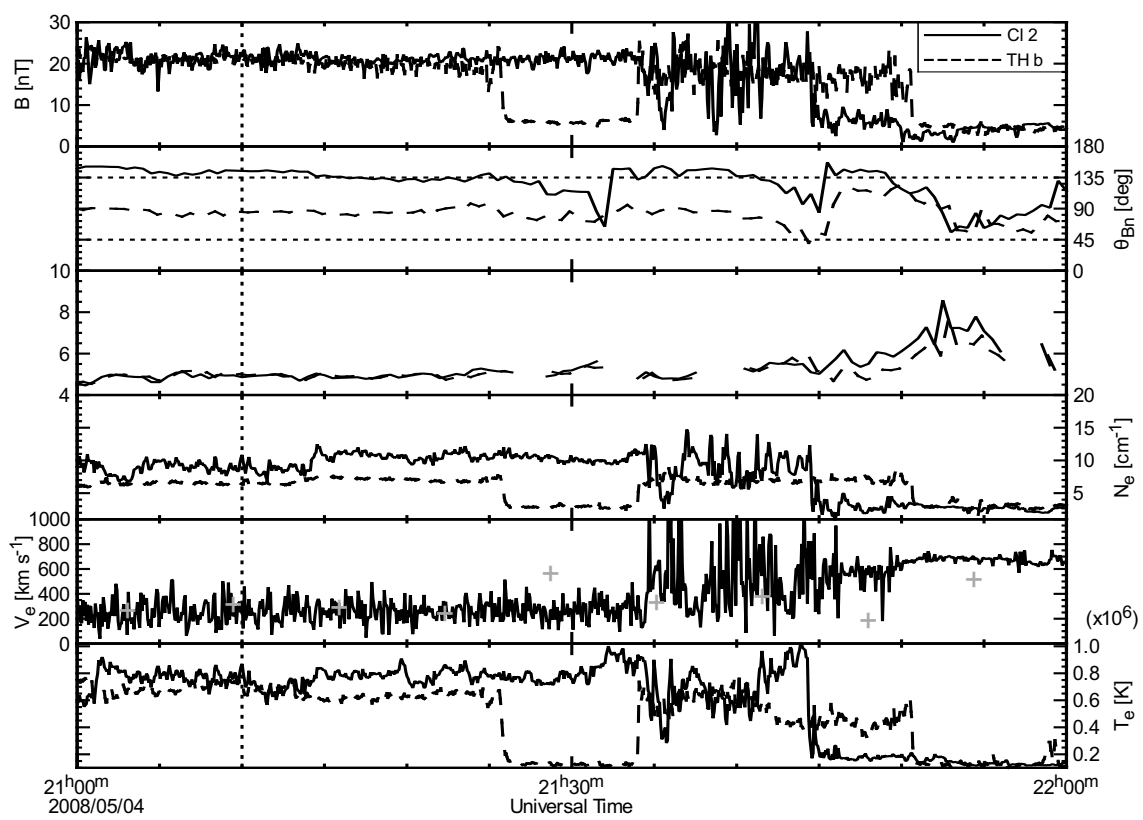

Fig. 1. Parameters measured by Cluster 2 and THEMIS b, and estimates of shock parameters upstream of the spacecraft locations using time-lagged data from ACE: (a) magnetic field, (b) $\theta_{\mathrm{Bn}}$, (c) the magnetosonic Mach number $M_{\mathrm{ms}}$, (d) electron number density, (e) electron bulk velocity (plus signs denote data from THEMIS b), and (f) electron temperature. The horizontal dotted lines in panel $(\mathbf{b})$ are at $\theta_{\mathrm{Bn}}=45^{\circ}$ and $135^{\circ}$, the approximate boundaries between quasi-parallel and quasi-perpendicular shocks. The vertical dotted line lies at time 21:10 UT, which is the when the electron distributions used here were measured.

and Scudder, 1984; Scudder et al., 1986a,c), wave turbulence (Galeev, 1976), various micro-instabilities (Wu et al., 1984), and scattering of electron trajectories in static electromagnetic fields (Balikhin et al., 1993). Detailed studies of these mechanisms exist (Savoini and Lembege, 1994; Gedalin and Griv, 1999; Hull et al., 2001; Lembège et al., 2003). Furthermore, having crossed the bowshock, distributions may continue to evolve as electrons travel deeper into the magnetosheath, in particular showing a growing temperature anisotropy (Masood and Schwartz, 2008) due in part to unexplained increases in average perpendicular temperature.

Large scale magnetosheath properties have long been suspected of influencing electron distributions (Feldman et al., 1983). Electrons travel along magnetic field lines which meet the bowshock at locations which may be separated by large distances, where the shock conditions are different. Travel along magnetic field lines may have a number of effects on electron distributions. Feldman et al. (1983) suggested that the increasing separation of two points connecting the bowshock with the magnetic field may lead to cooling of electrons trapped in the magnetosheath by the electrostatic potential barriers at the bowshock. Masood and Schwartz (2008) suggested that the decrease in field-aligned suprathermal electrons could occur as electrons follow the magnetic field line and escape back into the solar wind. Furthermore, the whistler anisotropy instability may effect the evolution of electron distributions as they move deeper into the shock (Gary et al., 2005).
In this paper electron trajectories are mapped from the bowshock to both the Cluster and THEMIS spacecraft. For the first time, we show that features in the electron distribution such as asymmetries about the pitch angle $\alpha=90^{\circ}$ can successfully be explained by comparing these with the total (ions plus electrons) heating predicted by the RankineHugoniot relations at crossing locations. Distributions of electrons whose trajectories pass through (or close by) both spacecraft are also compared. Section 2 discusses the data sets (Sect. 2.1), gives a brief introduction to the RankineHugoniot problem (Sect. 2.2), and discusses the mechanism. Results are given in Sect. 3 where electron bowshock crossing locations are mapped. Distributions of electrons whose trajectories connecting both spacecraft are studied in Sect. 3.2, Rankine-Hugoniot predictions of total heating at bowshock crossing locations are related to distribution asymmetries in Sect. 3.3. Concluding remarks are given in Sect. 4.

\section{Background}

In this section we present a brief background. Section 2.1 discusses the data sets, while Sect. 2.2 contains a review of the Rankine-Hugoniot problem. The latter subsection also reviews the method used for determining electron trajectories. 


\subsection{Data sets}

This paper compares simultaneous observations from both the Cluster and THEMIS spacecraft. The data sets come from the time interval 21:00 to 22:00 UT on 4 May 2008. Figure 1 shows parameters measured by the spacecraft during this interval, along with shock parameters estimated using data from the ACE spacecraft. During this period the Cluster 2 spacecraft and THEMIS b both perform an outward bound bowshock crossing. Note that due to the spacecraft position and motion of the bowshock, THEMIS $b$ reenters the magnetosheath at approximately $21: 34$, and performs a second outbound crossing at around 21:45 UT. Figure 2 shows projections of the positions of Cluster 2 and THEMIS $\mathrm{b}$ in the GSE $\mathrm{x}-\mathrm{y}$ plane $\left[(9.8,-10.9,0.050) R_{\mathrm{E}}\right.$ and $(5.9,16.8,-7.3) R_{\mathrm{E}}$, respectively, at $\left.21: 10 \mathrm{UT}\right]$, along with nominal positions for the bowshock and magnetopause. The surfaces are calculated using the method described by Schwartz (1998), using the parameters determined by Slavin and Holzer (1981) and Roelof and Sibeck (1993), respectively.

Electron data from the Cluster spacecraft is provided by the PEACE (Plasma Electron and Current Experiment) instrument (Johnstone et al., 1997). Each Cluster spacecraft contains two PEACE instruments giving a full $4 \pi$ solid angle view each spin, with an energy range of $0.7 \mathrm{eV}$ to $26 \mathrm{keV}$ and time resolution of $4 \mathrm{~s}$. Distributions plotted in this paper are corrected for the spacecraft potential, averaged over 5 spins (20 s) to improve distribution statistics, and re-binned over 13 pitch angles. PEACE calibrations yield moments accurate to $\sim 5 \%$, and counting statistics together with the standard deviation in 5-spin averages are insignificant. Therefore errors in phase-space distributions are smaller than the differences shown in the figures that follow. For a more detailed discussion of calibration see (Fazakerley et al., 2010). Magnetic field measurements are made using the Cluster FGM (Fluxgate Magnetometer) (Balogh et al., 1997).

The ESA (Electrostatic Analyser) (McFadden et al., 2008) is used to provide electron data from the THEMIS spacecraft. Each ESA instrument provides a full $4 \pi$ solid angle view with each spacecraft rotation over an energy range of $1.6 \mathrm{eV}$ to $25 \mathrm{keV}$. Electron data is gathered over 1 spacecraft spin period ( $3 \mathrm{~s})$, has been corrected for spacecraft potential, and re-binned over 13 pitch angles. Comparison of THEMIS and Cluster electron phase space distributions in the solar wind show close agreement, with differences smaller than those displayed in the following figures. Magnetic field measurements are made using the THEMIS FGM (Auster et al., 2008).

The upstream solar wind parameters used here are obtained from the ACE (Advanced Composition Explorer) spacecraft which sits in the solar wind upstream of the bowshock. Ion moments including mass density, flow velocity, and temperature are obtained from the SWEPAM (Solar Wind Electron Proton Alpha Monitor) experiment (Mc-

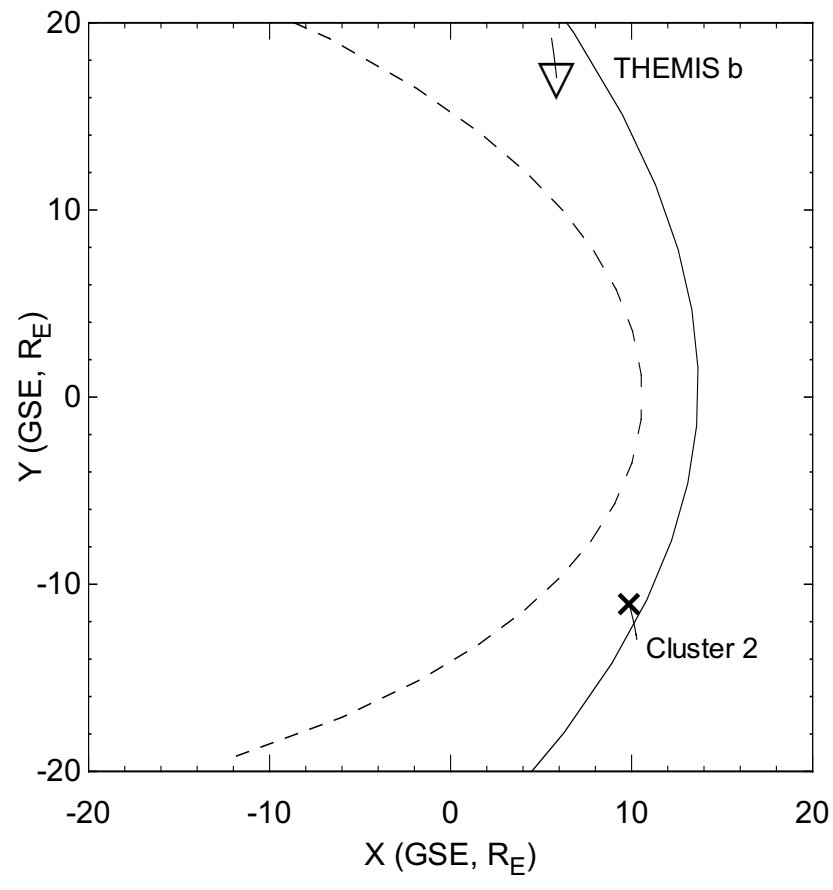

Fig. 2. Spacecraft trajectories: THEMIS b (Triangle) and Cluster 3 (cross) from 20:30 to 23:00 UT, 4 May 2008, along with nominal bowshock (solid) and magnetopause (dashed) locations. At $21: 10$ UT the spacecraft were located at $(9.8,-10.9,0.050)_{\mathrm{GSE}} R_{\mathrm{E}}$ and $(5.9,16.8,-7.3)_{\mathrm{GSE}} R_{\mathrm{E}}$, respectively.

Comas et al., 1998). The upstream magnetic field is determined using ACE's Magnetic Fields Experiment (Smith et al., 1998).

Of course, the time taken for plasma to propagate from one spacecraft to the next is not negligible. Therefore, to ensure that we are comparing similar conditions at each spacecraft we must take this propagation time into account. To find the appropriate time lag between these spacecraft we match features observed in the magnetic field traces. We find that ACE sees features in the magnetic field $2379 \mathrm{~s}$ prior to Cluster 2. Between Cluster 2 and THEMIS $b$ there is a further delay of $22 \mathrm{~s}$. As a result, data from Cluster 2 have been shifted forwards by $22 \mathrm{~s}$. For example in Fig. 1 the Cluster 2 trace has been moved $22 \mathrm{~s}$ to the right. Likewise, data from ACE used to calculate $\theta_{\mathrm{Bn}}$ and $M_{\mathrm{ms}}$ has been shifted $2391 \mathrm{~s}$.

\subsection{Rankine-Hugoniot relations}

The Rankine-Hugoniot relations allow the fluid properties downstream from a shock (the total fluid mass density, bulk flow velocity, and thermal pressure, along with the magnetic field) to be determined given the upstream quantities. Many texts deal with the Rankine-Hugoniot relations, for example Burgess (1995). The problem is typically formulated in terms of the (upstream) Alfvén Mach number $M_{\mathrm{A}}, \theta_{\mathrm{Bn}}$, which is the angle between the magnetic field $\boldsymbol{B}$ and the shock normal $\hat{\boldsymbol{n}}$, 


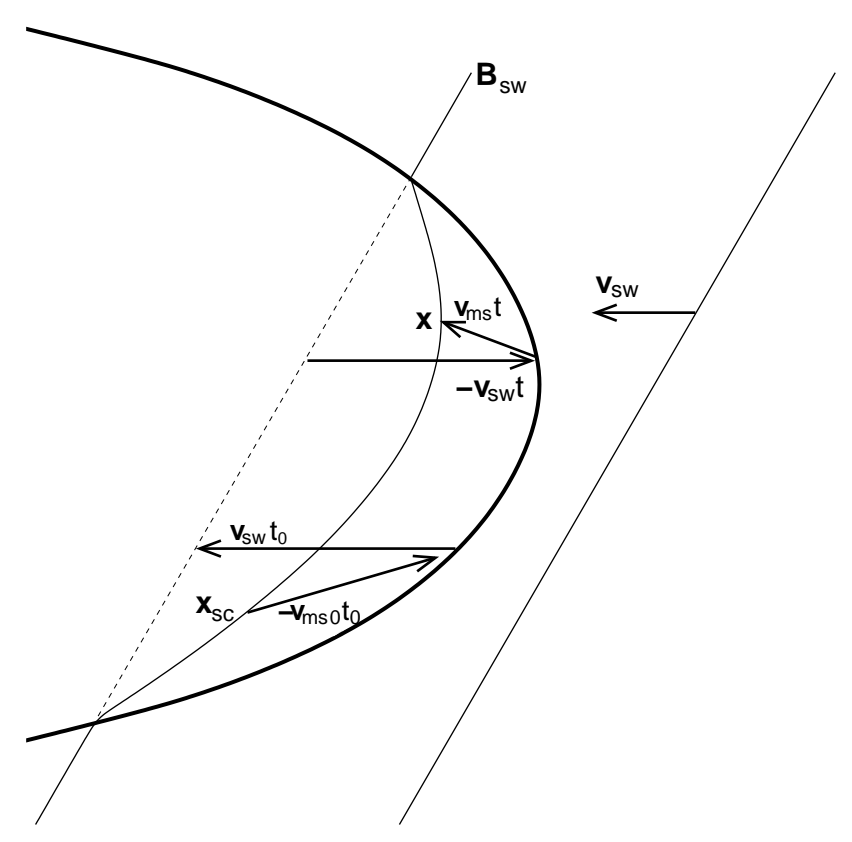

Fig. 3. Schematic demonstrating the geometry used to estimate electron trajectories inside the magnetosheath.

and the ratio $\beta$ between the thermal and magnetic pressures. In this paper we are particularly interested in the downstream temperature $T_{\mathrm{D}}=P_{\mathrm{D}} /\left(n_{\mathrm{D}} k_{\mathrm{B}}\right)$, where the subscript " $\mathrm{D}$ " denotes a downstream value, $n$ is the number density, $k_{\mathrm{B}}$ is the Boltzmann constant, and $P$ is the thermal pressure, for which the Rankine-Hugoniot relations predict

$$
\begin{aligned}
\frac{P_{\mathrm{D}}}{\rho_{\mathrm{U}} V_{\mathrm{U}}^{2}} & =\left(1-\frac{1}{r}\right)+\frac{P_{\mathrm{U}}}{\rho_{\mathrm{U}} V_{\mathrm{U}}^{2}} \\
& +\frac{\cos ^{2} \theta_{\mathrm{Bn}}}{2 M_{\mathrm{A}}^{2}}\left[1-\frac{\left(1-M_{\mathrm{A}}^{2} \sec ^{2} \theta_{\mathrm{Bn}}\right)^{2}}{\left(1-M_{\mathrm{A}}^{2} \sec ^{2} \theta_{\mathrm{Bn}} / r\right)^{2}}\right] .
\end{aligned}
$$

Here, the subscript "U" denotes an upstream value, $\rho$ is the mass density, $V$ is the component of the bulk velocity parallel to the shock normal, $v_{\mathrm{A}}$ and $M_{\mathrm{A}}$ are the Alfvén speed and Mach number, respectively, and $r=\rho_{\mathrm{D}} / \rho_{\mathrm{U}}$. Although the Rankine-Hugoniot relations constrain the total fluid density, velocity and pressure (and hence temperature) downstream of the shock, they do not treat individual species, specifically ions species and electrons, separately. Hence the fraction of the total heating taken up by electrons is not determined by the Rankine-Hugoniot relations, and depends instead upon the details of the physical processes taking place at the shock. The ratio of electron heating to total heating $\Delta T_{\mathrm{e}} / \Delta T_{\text {tot }}$ is approximately proportional to the inverse of the Alfvén Mach number $M_{\mathrm{A}}$ (Schwartz et al., 1988, Fig. 6a). Approximating a straight line fit for the data in that figure gives

$$
\frac{\Delta T_{\mathrm{e}}}{\Delta T_{\mathrm{tot}}} \approx \frac{1.2}{M_{\mathrm{Au}}}
$$

which may be used to predict empirically the electron heating. Typically, the fraction electron to total heating lies between 0.1 and 0.4 (Schwartz et al., 1988).

\subsection{Electron trajectories}

Electron trajectories within the magnetosheath studied in this paper are determined using a number of simplifications. A schematic diagram to illustrate the method used is shown in Fig. 3. First, we assume that the plasma seen by a spacecraft at position $\boldsymbol{x}_{\mathrm{sc}}$ has flowed in a straight line from some position on the bowshock to the spacecraft with a constant velocity $\boldsymbol{V}_{\mathrm{ms} 0}$, (the magnetosheath flow velocity). We use ion data from the spacecraft to determine $\boldsymbol{V}_{\mathrm{ms} 0}$ and then cross-check for consistency with the Rankine-Hugoniot relations at the shock, given solar wind values from ACE. We therefore trace along the negative velocity direction until the bowshock is reached at position $\boldsymbol{x}_{\mathrm{sc}}-\boldsymbol{V}_{\mathrm{ms} 0} t_{0}$, which defines the time $t_{0}$ taken by the parcel of plasma (and hence the field line) to reach the spacecraft. Changing position once again by adding $\boldsymbol{V}_{\text {sw }} t_{0}$, where $\boldsymbol{V}_{\mathrm{sw}}$ is the solar wind velocity, takes us to a position $\boldsymbol{x}_{\mathrm{sc}}+\left(\boldsymbol{V}_{\mathrm{sw}}-\boldsymbol{V}_{\mathrm{ms} 0}\right) t_{0}$. This new point is the location that the magnetic field line would have lain had it been undisturbed by the solar wind (the dashed line in Fig. 3). Any point on this hypothetical field line can be reached by moving parallel to the solar wind magnetic field. For our purposes, we divided the hypothetical (dashed) line into $N$ equally spaced points. From each of these points we move in the $-\boldsymbol{V}_{\text {sw }}$ direction until the bowshock is reached at a distance of $V_{\mathrm{sw}} t$ which defines $t$. Using the RankineHugoniot predictions, along with ACE observations to give the upstream conditions, we find the magnetosheath bulk velocity $\boldsymbol{V}_{\mathrm{ms}}$ and magnetic field $\boldsymbol{B}_{\mathrm{ms}}$. Again we assume that the plasma moves with a constant velocity (and with unchanging $\left.\boldsymbol{B}_{\mathrm{ms}}\right)$ a distance $\boldsymbol{V}_{\mathrm{ms}} t$ away from the shock to the point $\boldsymbol{x}$. This method allows us to find a locus of points $\boldsymbol{x}$ giving the approximate location of the field line, the magnetic field $\boldsymbol{B}(\boldsymbol{x})$, bulk velocity $\boldsymbol{V}_{\mathrm{ms}}(\boldsymbol{x})$, and the time $t(\boldsymbol{x})$ elapsed since the parcel of plasma crossed the bowshock, at a series of points along the field line. For the portion of the magnetosheath relatively close to the bowshock this method gives a simple but effective means to approximate the field lines along which electrons traverse the magnetosheath.

We assume that electron gyrocenters move along field lines with a velocity $v_{\|}$which varies such that the kinetic energy and first adiabatic invariant are conserved (thus taking into account of any magnetic mirroring). Motion perpendicular to the local magnetic field we assume to be due to $\boldsymbol{E} \times \boldsymbol{B}$ drift, with a drift velocity $\boldsymbol{v}_{\mathrm{D}}=\boldsymbol{V}_{\mathrm{ms}}-\left(\boldsymbol{B} \cdot V_{\mathrm{ms}}\right) \boldsymbol{B} / B^{2}$. Curvature and $\nabla B$ drifts are small and therefore are neglected. The crossing point of an electron with energy $E$ and pitch angle $\alpha$ can therefore be determined by moving backwards along the electron trajectories until the bowshock is reached. 


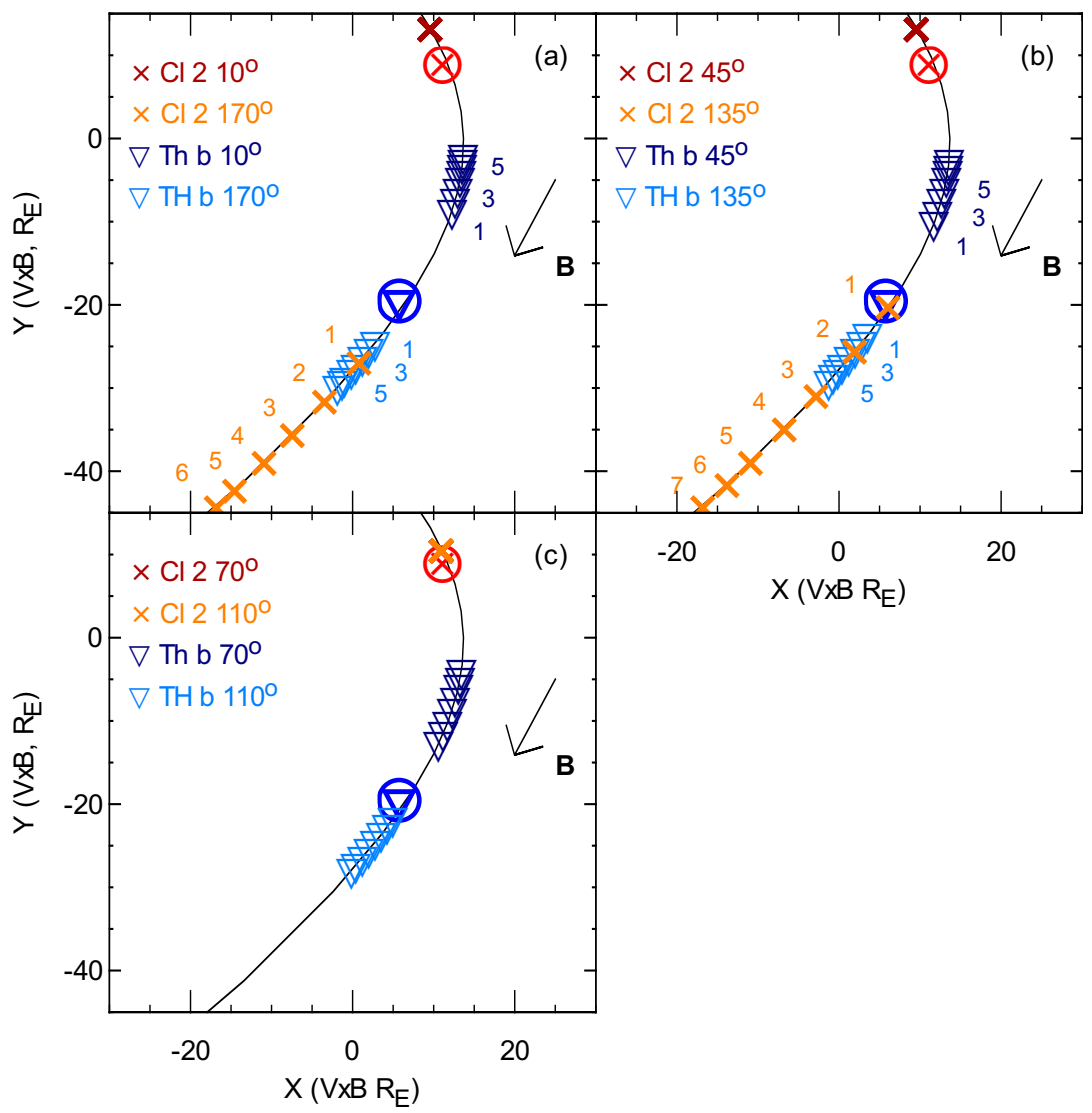

Fig. 4. Points on the bowshock at which electrons cross in order to reach either THEMIS b (encircled triangle) or Cluster (encircled cross). Electrons crossing at triangles travel to THEMIS b, while those crossing at crosses arrive at Cluster 2. The two angles shown are chosen so that all electrons have equal $\left|v_{\|}\right|=v|\cos \alpha|$. Crossing points are labelled 1 to 8, corresponding to energies 10, 16.7, 27.8, 46.4, 77, 129.2, 215.4 and $359.4 \mathrm{eV}$.

\section{Results}

In this section we examine the electrons that arrive at the Cluster and THEMIS spacecraft in the magnetosheath. Before looking in detail at the electron distributions in Sects. 3.2 and 3.3 we first study the locations at which electrons with given energies and pitch angles must cross the bowshock in order to reach the spacecraft.

\subsection{Bowshock crossing locations for electrons}

The figures in this section that show spacecraft positions and electron trajectories are in the $\boldsymbol{V} \times \boldsymbol{B}$ plane defined as follows: the z-axis is parallel to the vector $\boldsymbol{V} \times \boldsymbol{B}$ and is normal to the plane. The $x$-direction is chosen such that the GSE $x$ axis lies in the $x-z$ plane, and the $y$-axis is chosen to make the system right handed. The projection of the position of Earth onto the $\boldsymbol{V} \times \boldsymbol{B}$ plane determines the origin $(x=y=0)$. The plane chosen contains the THEMIS $b$ spacecraft. Note that at the time being examined in this paper (21:00 to 22:00 UT on 4 May 2008) both THEMIS $b$ and Cluster 2 are in approximately the same $\boldsymbol{V} \times \boldsymbol{B}$ plane.
Figure 4 shows the position at which electrons with pitch angles $\alpha$ for several energies crossed the bowshock in order to reach either the Cluster or the THEMIS spacecraft. Figure 4a displays bowshock crossing points for electrons with pitch angles $\alpha=10^{\circ}$ and $170^{\circ}$, Fig. $4 \mathrm{~b}$ for $\alpha=45^{\circ}$ and $135^{\circ}$, and Fig. $4 \mathrm{c}$ for $\alpha=70^{\circ}$ and $110^{\circ}$. Note that for each plot the two angles both have the same value of $\left|90^{\circ}-\alpha\right|$. This means that for a given kinetic energy the pitch angles correspond to $v_{\|}$with equal magnitudes but opposite signs.

For electrons travelling to each spacecraft, and for each value of $\alpha, 8$ points are displayed, corresponding to different kinetic energies. The eight values of kinetic energy are 10.0, 16.7, 27.8, 46.4, 77.4, 129, 215.4 and $359.4 \mathrm{eV}$. Crossings for electrons with higher kinetic energy tend to be further from the spacecraft since these electrons have higher $v_{\|}$in relation to the $\boldsymbol{E} \times \boldsymbol{B}$ drift. A typical value for the electron temperature in the magnetosheath is $T_{\mathrm{e}} \sim 8 \times 10^{5} \mathrm{~K}$, which is equivalent to $\sim 70 \mathrm{eV}$. Thus, the spread of energies chosen here covers the thermal range well.

At the time of interest (21:10 UT, the vertical dotted line in Fig. 1) THEMIS b lies behind a quasi-perpendicular shock, 


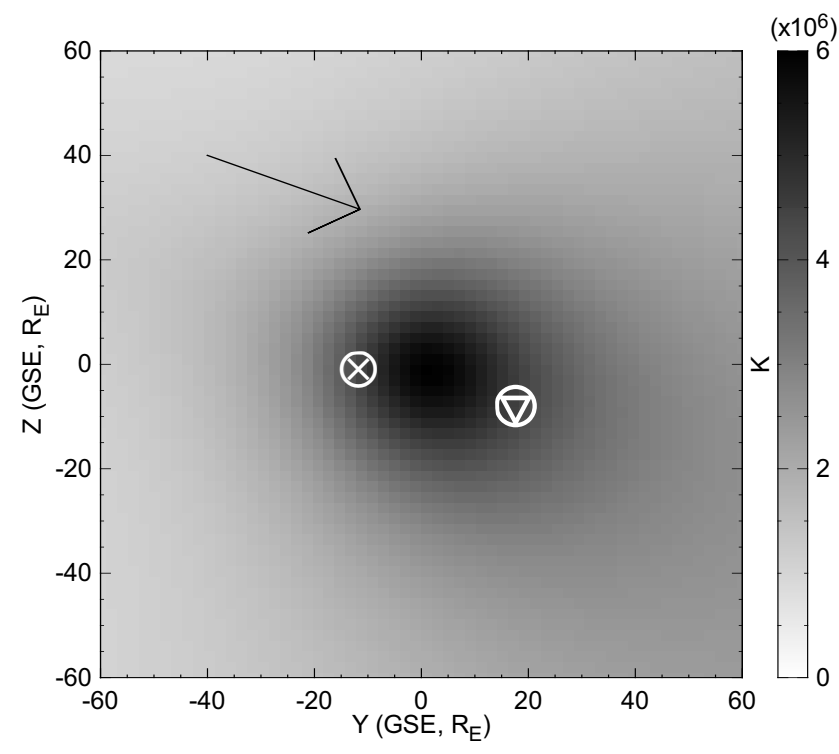

Fig. 5. A map of the Rankine-Hugoniot predicted total temperature on the bowshock surface. The upstream parameters $(\boldsymbol{B}, \boldsymbol{V}, \rho$, and $T$ ) are obtained from the ACE spacecraft. The circled triangle and cross show the position of THEMIS $b$ and Cluster 2, while the arrow denotes the upstream magnetic field direction in the $y$-z plane.

with $\theta_{\mathrm{Bn}}=84.3^{\circ}, M_{\mathrm{A}}=6.7$, and $M_{\mathrm{ms}}=5.8$. As a result, electrons moving along magnetic field lines travel approximately parallel to the shock surface. Thus electrons arriving at THEMIS $\mathrm{b}$ with pitch angle $\alpha<90^{\circ}$, and those with $\alpha>90^{\circ}$ lie in two different camps on the bowshock surface on either side of the spacecraft. For this particular geometry, we find electrons with $v_{\|}>0\left(\alpha<90^{\circ}\right)$, lie very close to the bowshock nose where $\left|V_{\mathrm{n}}\right|$, and therefore $M_{\mathrm{ms}}$, are maximized. The Rankine-Hugoniot relations predict that the total heating in this region of the shock is at its largest (Fig. 5). On the other hand, electrons with $v_{\|}<0\left(\alpha>90^{\circ}\right)$ originate further down the flank, where the total heating is lower.

The quasi-parallel shock geometry near the Cluster 2 spacecraft $\left(\theta_{\mathrm{Bn}}=158.1^{\circ}, \quad M_{\mathrm{A}}=6.3\right.$, and $\left.M_{\mathrm{ms}}=6.1\right)$ presents quite a different scenario. Electrons with $\alpha<90^{\circ}$ enter the magnetosheath very near to the spacecraft, and the distance travelled to reach the spacecraft from the bowshock is very small. This leads to a very small spread in bowshock crossing positions for $\alpha<90^{\circ}$ electrons. Conversely, electrons with $\alpha>90^{\circ}$ must travel large distances through the magnetosheath before they encounter the spacecraft, leading to a very large spread in crossing positions. In this geometry most electrons do not suffer the maximal heating at the bowshock nose, tending instead to originate at the flanks of the bowshock. Exceptions to this exist for electrons with $\alpha \gtrsim 45^{\circ}$ and low energies, which originate near the bowshock nose.

One important observation is that a significant overlap exists between bowshock crossing regions for electrons arriving at THEMIS b, and for electrons arriving at Cluster with $\alpha>90^{\circ}$. We may expect that the velocity distributions for these electrons measured by these spacecraft could show significant similarities, but only if the electrons travel more or less kinematically, that is, there is no significant influence due to effects such as instabilities. These distributions will be studied in Sect. 3.2.

\subsection{THEMIS and Cluster electron distributions}

In this subsection we study electron pitch angle distributions measured by Cluster, $f_{\mathrm{C}}(E, \alpha)$, and THEMIS b, $f_{\mathrm{T}}(E, \alpha)$. In particular we examine the distributions of electrons whose pitch angles and energy ranges suggest a common bowshock crossing point, based on the analysis shown in Fig. 4.

Figure 6 shows electron phase space distributions at a range of pitch angles $\alpha>90^{\circ}$, chosen to approximately match the pitch angles studied in Fig. 4 for both Cluster 2 and THEMIS $b$. We see that the distributions measured by both spacecraft match quite closely. Since these electrons have similar crossing points, it follows that trajectories reaching Cluster 2 pass close to THEMIS b. The similarity of the THEMIS and Cluster electron distributions therefore suggests that the electrons travel more or less kinematically across the magnetosheath, and that no significant heating of the electrons has taken place during their travel.

Crossing points for electrons with $\alpha \sim 90^{\circ}$ are displayed in Fig. 4c. Here, both $v_{\|}>0$ and $v_{\|}<0$ electrons reaching Cluster originate from very similar spots on the bowshock. This is due to the increase in magnetic field strength along the field line close to Cluster which causes magnetic mirroring of the electrons, which then stream back towards the spacecraft with a reversed pitch angle $\alpha \rightarrow 180^{\circ}-\alpha$. Therefore, one can expect to find that

$f_{\mathrm{SC}}(E, \alpha) \approx f_{\mathrm{SC}}\left(E, 180^{\circ}-\alpha\right)$,

when $\alpha$ is close to $90^{\circ}$. Such symmetric distributions are observed for Cluster 2 in Fig. 6c, in which both traces for Cluster 2 lie almost on top of each other. The traces observed by THEMIS $b$ also show a large degree of symmetry in Fig. 6c. As may be seen in that panel, and as is commonly found in the magnetosheath, distributions of electrons with $\alpha \approx 90^{\circ}$ tend to be smoother and than for other pitch angles. We therefore conclude that electron heating information is not well retained for electron distributions where $\alpha \approx 90^{\circ}$.

\subsection{Comparison of Rankine-Hugoniot predictions with spacecraft data}

In this subsection we look in more detail at the relationship between the electron distributions observed by the spacecraft and the Rankine-Hugoniot total heating requirements at the locations where the electrons crossed the bowshock. Figure 7 displays electron phase space density distributions for pitch angle pairs $\alpha$ and $180^{\circ}-\alpha$. As in Fig. 6 the solid and dashed traces refer to Cluster 2 and THEMIS b, respectively. 


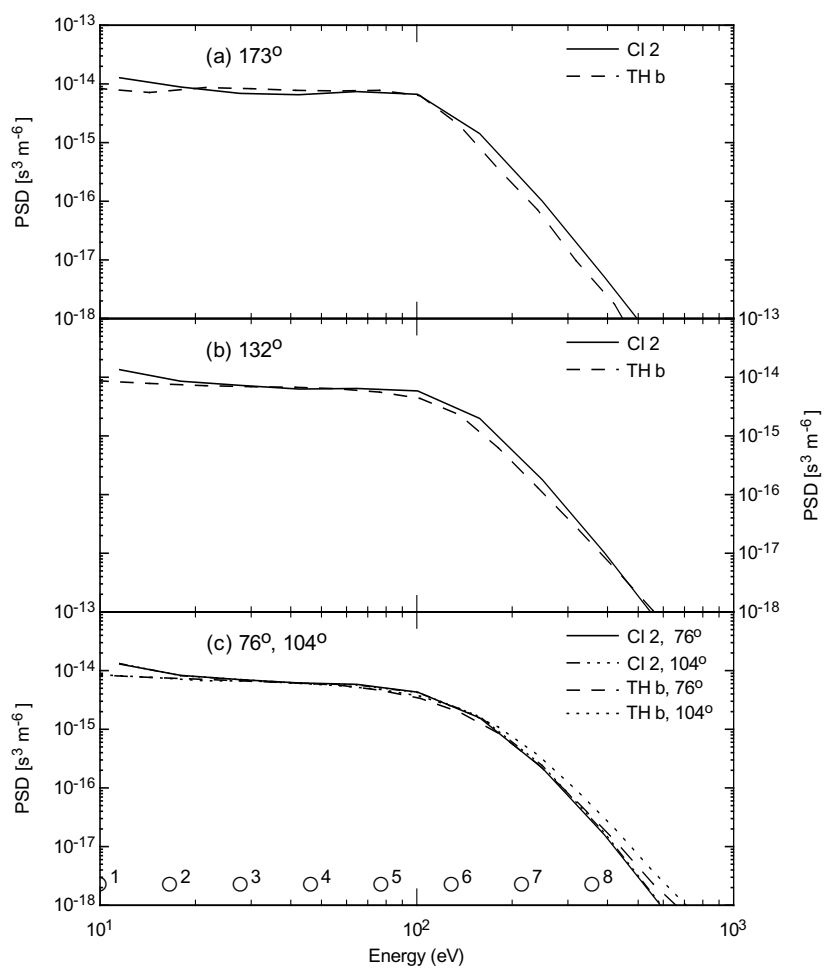

Fig. 6. Electron distributions for $\alpha>90^{\circ}\left(v_{\|}<0\right)$. Additionally, panel (c) also shown distributions for $\alpha<90^{\circ}$. The overlap between distributions seen by Custer 2 and THEMIS $b$ is related to the similar bowshock crossing points for electrons arriving at each spacecraft. This is particularly true for low energy electrons. The circles in panel (c) correspond to the energies shown in Fig. 4.

We immediately see that the distribution measured by THEMIS $b$ is highly asymmetric for pitch angles far from $90^{\circ}$. This feature is easily understood in a qualitative sense simply by referring to Fig. 4 . Figure 8 shows shock parameters and predictions of the Rankine-Hugoniot relations calculated on the cut of the bowshock surface displayed in Fig. 4 . The predicted downstream total temperature is clearly greater by a factor of $\sim 2$ at the nose than on the flanks. The temperature of crossing points can be seen by comparing the y-coordinates in Fig. 4 with the horizontal axis in this figure. We find that electrons reaching THEMIS $\mathrm{b}$ with $\alpha<90^{\circ}$ originate from locations close to the bowshock nose where total heating is greatest, so we expect the source distribution of these electrons to be hotter than the source of the $\alpha>90^{\circ}$ pitch angle electrons. This provides a qualitative explanation for the asymmetric distributions observed by THEMIS $b$. Below we discuss the influence of $M_{\mathrm{A}}$ in Eq. (2) on the fraction of total heating taken up by the electrons.

In order to assess the contributions made to the electron temperature by electrons arriving from different crossing locations we define the following quantities, which measure the contribution of particles of pitch angle $\alpha$ to the parallel and perpendicular temperature:

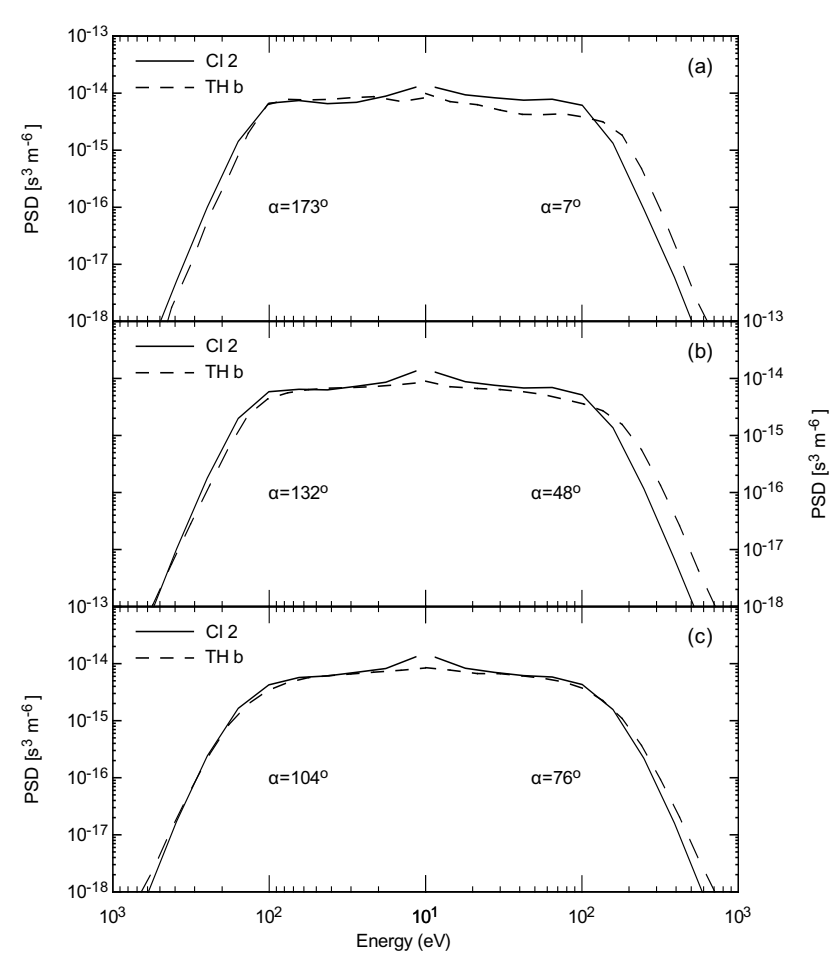

Fig. 7. Electron distributions measured at Cluster 2 and THEMIS $b$. Positive $v_{\|}$electron distributions $\left(\alpha<90^{\circ}\right)$ are on the right, negative $v_{\|}$are on the left. The hotter distributions for THEMIS b with $\alpha<$ $90^{\circ}$ arise due larger to electron crossing locations for such electrons.

$\delta T_{\|}(\alpha)=\frac{2 \pi m_{\mathrm{e}}}{n_{\mathrm{e}} k_{\mathrm{B}}} \sin \alpha \cos ^{2} \alpha \delta \alpha \int_{0}^{\infty} d v v^{2} f[E(v), \alpha]$,

and

$\delta T_{\perp}(\alpha)=\frac{\pi m_{\mathrm{e}}}{n_{\mathrm{e}} k_{\mathrm{B}}} \sin ^{3} \alpha \delta \alpha \int_{0}^{\infty} d v v^{2} f[E(v), \alpha]$,

where $m_{\mathrm{e}}$ is the electron mass, $n_{\mathrm{e}}$ is the number density, and $k_{\mathrm{B}}$ is the Boltzmann constant. Integrating Eqs. (4) and (5) over $\alpha$ therefore gives $T_{\|}$and $T_{\perp}$, respectively. We define $T_{\| \pm}$by integrating $\delta T_{\|}(\alpha)$ from $\alpha=0^{\circ}$ to $90^{\circ}$ (+ sign), or from $90^{\circ}$ to $180^{\circ}$ ( - sign), with $T_{\perp \pm}$ defined in an analogous way. Partial temperatures calculated in this manner are contained in Table 1, and plots of $\delta T_{\|}$and $\delta T_{\perp}$ are displayed in Fig. 9. The asymmetry is immediately obvious for THEMIS $\mathrm{b}$, with $T_{\|+}>T_{\|-}$being particularly strong. An opposite, but weaker asymmetry is seen in $T_{\|}$for Cluster 2 . Similar, opposite asymmetries for $T_{\perp}$ are evident in Fig. 9, although these are weaker still.

Table 2 shows the bulk temperature predicted by the Rankine-Hugoniot relations at bowshock crossing locations for electrons which reach the spacecraft with energy $E$ and $\alpha=35^{\circ}$ and $145^{\circ}$, (since electrons with pitch angles around these values contribute most to $T_{\|}$, as may be seen from Fig. 9) along with $M_{\mathrm{A}}$. The Rankine-Hugoniot relations do not treat electron and ion species separately, so we have 
Table 1. Temperature contributions, $T_{+}$and $T_{-}\left(10^{6} \mathrm{~K}\right)$ calculated by integrating Eqs. (4) and (5) over pitch angles $\alpha$. $T_{\text {tot }}$ is $\frac{1}{3}\left(T_{\|}+\right.$ $\left.2 T_{\perp}\right)$.

\begin{tabular}{ccccc}
\hline$\sigma$ & $T_{\sigma}$ & $T_{\sigma+}$ & $T_{\sigma-}$ & $T_{\sigma+} / T_{\sigma-}$ \\
\hline \multicolumn{5}{c}{ THEMIS b } \\
\hline$\|$ & 0.823 & 0.475 & 0.349 & 1.36 \\
$\perp$ & 0.775 & 0.496 & 0.417 & 1.19 \\
tot & 0.791 & 0.489 & 0.394 & 1.24 \\
\hline \multicolumn{5}{c}{ Cluster 2} \\
\hline$\|$ & 0.773 & 0.363 & 0.411 & 0.88 \\
$\perp$ & 0.752 & 0.430 & 0.456 & 0.94 \\
tot & 0.759 & 0.408 & 0.441 & 0.93 \\
\hline
\end{tabular}

Table 2. Rankine-Hugoniot total temperatures (in units of $10^{6} \mathrm{~K}$ ), Mach numbers and electron temperature ratios at bowshock crossing points corresponding to different energy and pitch angle electrons arriving at the position of THEMIS $b$ and Cluster 2 .

\begin{tabular}{lcccccc}
\hline$E(\mathrm{eV})$ & 10 & 16.7 & 27.8 & 46.4 & 77.4 & 129.2 \\
\hline \multicolumn{7}{c}{ THEMIS b } \\
\hline$T_{\mathrm{RH}}\left(E, 35^{\circ}\right)$ & 5.35 & 5.48 & 5.62 & 5.72 & 5.80 & 5.85 \\
$T_{\mathrm{RH}}\left(E, 145^{\circ}\right)$ & 3.98 & 3.91 & 3.83 & 3.77 & 3.70 & 3.66 \\
$M_{\mathrm{A}}\left(E, 35^{\circ}\right)$ & 5.99 & 6.05 & 6.12 & 6.14 & 6.17 & 6.18 \\
$M_{\mathrm{A}}\left(E, 145^{\circ}\right)$ & 5.28 & 5.24 & 5.20 & 5.16 & 5.12 & 5.09 \\
$T_{\mathrm{e}}\left(E, 35^{\circ}\right) /$ & 1.17 & 1.20 & 1.23 & 1.26 & 1.28 & 1.29 \\
$T_{\mathrm{e}}\left(E, 145^{\circ}\right)$ & & & & & & \\
\hline & & & & & \\
\hline$T_{\mathrm{RH}}\left(E, 35^{\circ}\right)$ & 3.91 & 3.91 & 3.91 & 3.91 & 3.91 & 3.91 \\
$T_{\mathrm{RH}}\left(E, 145^{\circ}\right)$ & 4.27 & 3.83 & 3.48 & 3.28 & 3.12 & 3.02 \\
$M_{\mathrm{A}}\left(E, 35^{\circ}\right)$ & 5.99 & 6.05 & 6.12 & 6.14 & 6.17 & 6.18 \\
$M_{\mathrm{A}}\left(E, 145^{\circ}\right)$ & 5.28 & 5.24 & 5.20 & 5.16 & 5.12 & 5.09 \\
$T_{\mathrm{e}}\left(E, 35^{\circ}\right) /$ & 0.82 & 0.90 & 0.96 & 1.01 & 1.04 & 1.07 \\
$T_{\mathrm{e}}\left(E, 145^{\circ}\right)$ & & & & & & \\
\hline
\end{tabular}

$T_{\mathrm{RH}}=T_{\mathrm{i}}+T_{\mathrm{e}}$, which relates the Rankine-Hugoniot, ion and electron temperatures. The expected electron heating may be found using $\Delta T_{\mathrm{RH}}$ and $M_{\mathrm{A}}$ using Eq. (2). We can therefore assess the electron heating dependencies by comparing ratios of $T_{\mathrm{e}}(E, \alpha)$ at different crossing locations, or equivalently different energies and pitch angles (cf. Fig. 4) with ratios of $T_{+}$and $T_{-}$. Specifically, we compare the ratio $T_{+} / T_{-}$ (last column in Table 1) with $T_{\mathrm{e}}\left(E, 45^{\circ}\right) / T_{\mathrm{e}}\left(135^{\circ}\right)$ (Table 2), finding that for low energies these values match well.

To predict the asymmetry $T_{\|+} / T_{\|-}$using this method clearly one must carefully consider the contribution made by electrons originating from many bowshock crossing points. A simple estimate is made here by taking the average of the ratios $T_{\mathrm{e}}\left(E, 45^{\circ}\right) / T_{\mathrm{e}}\left(E, 135^{\circ}\right)$ for the first four energy values listed in Table 2. For THEMIS b the average is 1.24,

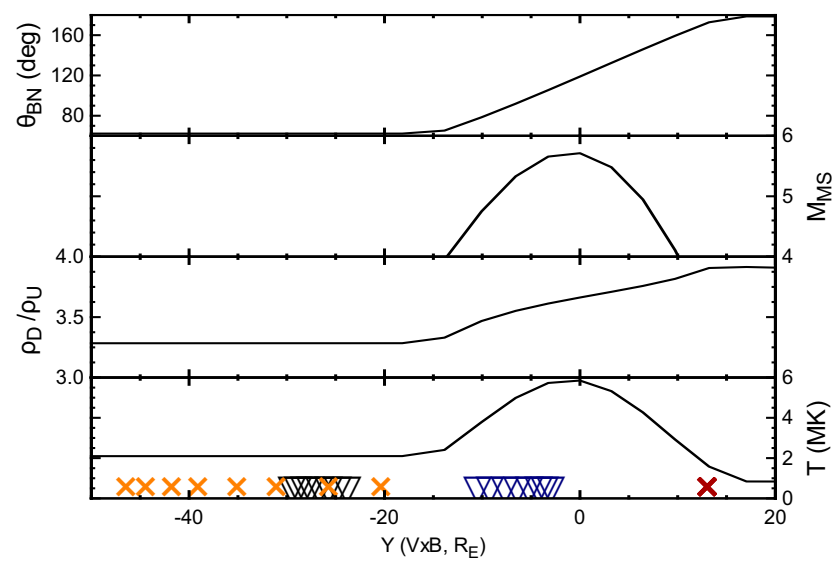

Fig. 8. Shock parameters and Rankine-Hugoniot predictions against bowshock $y_{V \times B}$ coordinate: (a) $\theta_{\mathrm{Bn}}$, (b) the magnetosonic Mach number $M_{\mathrm{ms}}$, (c) the ratio of downstream to upstream bulk density $\rho_{\mathrm{D}} / \rho_{\mathrm{U}}$, and (d) the downstream temperature. The triangles and crosses in (c) are correspond to those in Fig. 4b.

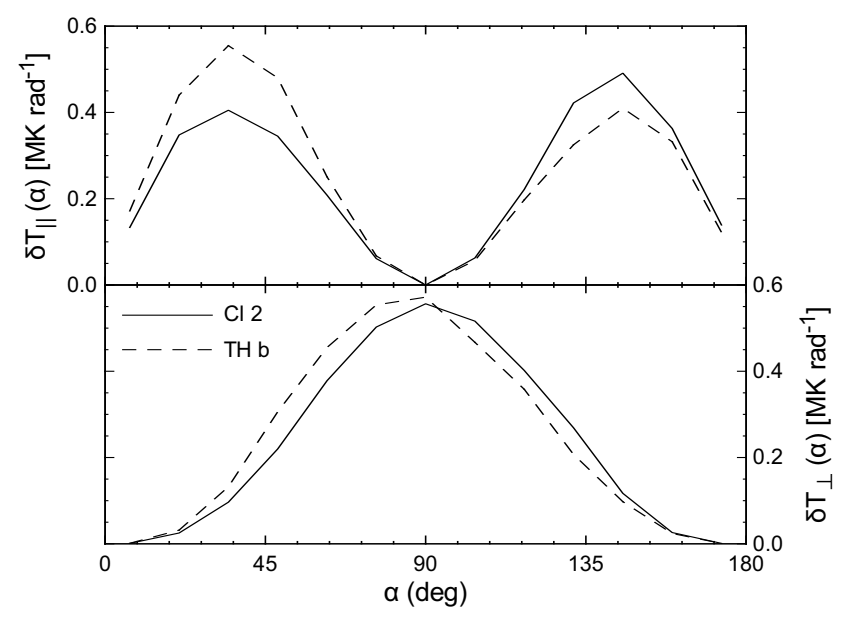

Fig. 9. Plot of $\delta T_{\|}(\alpha)$ and $\delta T_{\perp}(\alpha)$ defined by Eqs. (4) and (5) and show the contribution to the temperature moment of electrons as a function of $\alpha$. For $T_{\|}$the max is at $\sim 35^{\circ}$ and $\sim 145^{\circ}$. Asymmetries are especially visible for THEMIS $b$.

which compares reasonably well with the observed total and parallel asymmetries of 1.24 and 1.36. This origin of this asymmetry can be clearly observed in Fig. 7a and b, where the electrons observed by THEMIS $b$ extend further on the right side of the panel (corresponding to $\alpha<90^{\circ}$ ) than on the left. The average ratio $T_{\mathrm{e}}\left(E, 45^{\circ}\right) / T_{\mathrm{e}}\left(E, 135^{\circ}\right)$ for Cluster 2 is 0.97 , with the observed total and parallel asymmetries 0.93 and 0.88 . These asymmetries compare very favourably, and suggest that electron distributions retain much of the electron heating information from the electrons' bowshock crossing locations. 


\section{Conclusion}

The simultaneous positioning of both Cluster and THEMIS $b$ near to the Earth's bowshock, but widely separated from each other, along with a favourable orientation of the interplanetary magnetic field, has enabled us to study the manner in which magnetosheath electron distributions observed even close to the bowshock are a convolution of populations arriving from distant locations on the bowshock. Since the electron and ion heating are coupled through the local RankineHugoniot requirements and dependent upon, for example, the local cross shock potential, the imprint of distant locations on the electron populations on the electron population renders the bowshock heating a global problem. Distributions of incoming electron measured very close to the bowshock might be expected to be affected by heating at one very small region of the shock. However, out-going electrons are heated at distant bowshock locations. These electrons could plausibly affect the heating at the bowshock location where they exit the magnetosheath (e.g. by influencing the cross shock potential). This electron "cross-talk" may therefore render heating at the bowshock to be a global problem, rather than being influenced only by the local upstream conditions and shock geometry.

We find that velocity distributions of electrons which crossed the bowshock at closely spaced points on the bowshock show strong similarities when viewed from either the Cluster or THEMIS spacecraft. This is despite the fact that such electrons must travel large distances of $\sim 30 R_{\mathrm{E}}$ between these two spacecraft. We may therefore conclude that electrons travel somewhat kinematically within the bowshock, and that the electron distributions do not undergo major changes while travelling across the magnetosheath. Note that this may not be the case deeper in the magnetosheath.

We also studied the asymmetry of electron distributions, and compared them with the local Rankine-Hugoniot relations at the locations where the electrons first crossed the bowshock. We find that electrons travelling in opposite directions relative to the magnetic field often originate from widely separated positions on the bowshock, where the Rankine-Hugoniot total heating may differ by a factor of $\sim 2$. We then compared the ratio of the predicted electron temperatures at crossing locations, determined using Eq. (2) with $T_{\mathrm{RH}}$ and $M_{\mathrm{A}}$, for electrons with positive and negative $v_{\|}$with ratio of the temperatures calculated using electrons with positive and negative $v_{\|}$. We find that these ratios match closely for both spacecraft, especially when examining the heating at crossing points for low energy electrons. At higher energies however this ratio tends to move away from 1 , as the crossing points move down the flank with increasing energy. This remarkable match between the the ratio of the forward and backward moving electrons and the Rankine Hugoniot temperatures suggests that the electron distributions retain a memory of their various crossing locations, and the heating at those points, even though such electrons may have travelled very large distances. Furthermore, moments of the the electron distribution, such as the temperature, depend on shock properties at widely separated points, and as such are dependent on global bowshock properties.

This has a number of implications for electron heating at shocks. Because electrons retain a memory of the heating at their widely spaced crossing locations we conclude that the electron temperature, and in particular the parallel temperature, are affected by conditions over large areas of the shock surface. The perpendicular temperature is likely to be less affected since it is dominated by electrons with small $v_{\|}$ that may undergo magnetic mirroring multiple times meaning that their trajectories follow the bulk plasma flow more closely than electrons with larger $\left|v_{\|}\right|$. Furthermore, the contribution to the temperature of these electrons is proportional to the temperature predicted by the Rankine-Hugoniot relations at the electrons' crossing locations. This implies that in order to determine the partition of energy in heating the various species one must take into account the potentially large differences in heating experienced by electrons at any location in the magnetosheath, since this heating will depend strongly upon the electrons' energies and pitch angles.

Some remaining issues include the effects of internal magnetosheath processes, such as wave-particle instabilities. We note here that the electron trajectories follow closely the magnetosheath magnetic field lines, which here are close to the shock surface. Although such effects are shown to be small here it seems likely that deeper inside the sheath such effects may become more important. Furthermore, high energy electrons tend to travel much further inside the sheath due to their more distant crossing locations. These electrons may also be subject to instabilities and other effects. This may account for the temperature ratios diverging from 1 at higher energies.

Acknowledgements. This work relies on data from the Cluster Fluxgate Magnetometer team (E. Lucek, PI), and the Plasma Electron and Current Experiment (PEACE) electron team (A. N. Fazakerley). We acknowledge NASA contract NAS5-02099 and V. Angelopoulos for use of data from the THEMIS Mission. Specifically: C. W. Carlson and J. P. McFadden for use of ESA data, and K. H. Glassmeier, U. Auster and W. Baumjohann for the use of FGM data provided under the lead of the Technical University of Braunschweig and with financial support through the German Ministry for Economy and Technology and the German Center for Aviation and Space (DLR) under contract 50 OC 0302. We thank the ACE SWEPAM and MAG instrument teams and the ACE Science Center for providing the ACE data. Data analysis was done with the QM Science Analysis System for Cluster (QSAS) provided by the United Kingdom Cluster Science Centre (Imperial College, London, and Queen Mary, University of London). This work was funded by STFC.

Topical Editor R. Nakamura thanks M. Thomsen and another anonymous referee for their help in evaluating this paper. 


\section{References}

Auster, H. U., Glassmeier, K. H., Magnes, W., Aydogar, O., Baumjohann, W., Constantinescu, D., Fischer, D., Fornacon, K. H., Georgescu, E., Harvey, P., Hillenmaier, O., Kroth, R., Ludlam, M., Narita, Y., Nakamura, R., Okrafka, K., Plaschke, F., Richter, I., Schwarzl, H., Stoll, B., Valavanoglou, A., and Wiedemann, M.: The THEMIS Fluxgate Magnetometer, Space Sci. Rev., 141, 235-264, 2008.

Balikhin, M., Gedalin, M., and Petrukovich, A.: New mechanism for electron heating in shocks, Phys. Rev. Lett., 70, 1259-1262, 1993.

Balogh, A., Dunlop, M. W., Cowley, S. W. H., Southwood, D. J., Thomlinson, J. G., Glassmeier, K. H., Musmann, G., Luhr, H., Buchert, S., Acuna, M. H., Fairfield, D. H., Slavin, J. A., Riedler, W., Schwingenschuh, K., and Kivelson, M. G.: The Cluster Magnetic Field Investigation, Space Sci. Rev., 79, 65-91, 1997.

Burgess, D.: Collisionless shocks, in: Introduction to Space Physics, edited by: Kivelson, M. G. and Russell, C. T., pp. 129163, Cambridge University Press, Cambridge, UK, 1995.

de Hoffmann, F. and Teller, E.: Magneto-Hydrodynamic Shocks, Physical Rev., 80, 692-703, 1950.

Fazakerley, A. N., Lahiff, A. D., Rozum, I., Kataria, D., Bacai, H., Anekallu, C., West, M., and Åsnes, A.: Cluster-PEACE Inflight Calibration Status, pp. 281-299, 2010.

Feldman, W. C., Anderson, R. C., Bame, S. J., Gary, S. P., Gosling, J. T., McComas, D. J., Thomsen, M. F., Paschmann, G., and Hoppe, M. M.: Electron velocity distributions near the earth's bow shock, J. Geophys. Res., 88, 96-110, 1983.

Galeev, A. A.: Collisionless shocks, in: Physics of Solar Planetary Environments, edited by: Williams, D. J., pp. 464-490, 1976.

Gary, S. P., Lavraud, B., Thomsen, M. F., Lefebvre, B., and Schwartz, S. J.: Electron anisotropy constraint in the magnetosheath: Cluster observations, Geophys. Res. Lett., 32, 13109, doi:10.1029/2005GL023234, 2005.

Gedalin, M. and Griv, E.: Role of overshoots in the formation of the downstream distribution of adiabatic electrons, J. Geophys. Res., 104, 14821-14826, 1999.

Goodrich, C. C. and Scudder, J. D.: The adiabatic energy change of plasma electrons and the frame dependence of the cross-shock potential at collisionless magnetosonic shock waves, J. Geophys. Res., 89, 6654-6662, 1984.

Hull, A. J., Scudder, J. D., Larson, D. E., and Lin, R.: Electron heating and phase space signatures at supercritical, fast mode shocks, J. Geophys. Res., 106, 15711-15734, 2001.

Johnstone, A. D., Alsop, C., Burge, S., Carter, P. J., Coates, A. J., Coker, A. J., Fazakerley, A. N., Grande, M., Gowen, R. A., Gurgiolo, C., Hancock, B. K., Narheim, B., Preece, A., Sheather, P. H., Winningham, J. D., and Woodliffe, R. D.: Peace: a Plasma Electron and Current Experiment, Space Sci. Rev., 79, 351-398, 1997.

Kennel, C. F., Edmiston, J. P., and Hada, T.: A quarter century of collisionless shock research, Washington D.C. American Geophysical Union Geophysical Monograph Series, 34, 1-36, 1985.

Kivelson, M. G. and Russell, C.-T.: Introduction to Space Physics, edited by: Kivelson, M. G. and Russell, C. T., ISBN 0521451043, Cambridge, UK, Cambridge University Press, 1995.

Lembège, B., Savoini, P., Balikhin, M., Walker, S., and Krasnoselskikh, V.: Demagnetization of transmitted electrons through a quasi-perpendicular collisionless shock, J. Geophys. Res. (Space Physics), 108, 1256-1274, 2003.

Lembège, B., Giacalone, J., Scholer, M., Hada, T., Hoshino, M., Krasnoselskikh, V., Kucharek, H., Savoini, P., and Terasawa, T.: Selected Problems in Collisionless-Shock Physics, Space Sci. Rev., 110, 161-226, 2004.

Longmore, M., Schwartz, S. J., Geach, J., Cooling, B. M. A., Dandouras, I., Lucek, E. A., and Fazakerley, A. N.: Dawndusk asymmetries and sub-Alfvénic flow in the high and low latitude magnetosheath, Ann. Geophys., 23, 3351-3364, doi:10.5194/angeo-23-3351-2005, 2005.

Masood, W. and Schwartz, S. J.: Observations of the development of electron temperature anisotropies in Earth's magnetosheath, J. Geophys. Res. (Space Physics), 113, 1216, doi:10.1029/2007JA012715, 2008.

McComas, D. J., Bame, S. J., Barker, P., Feldman, W. C., Phillips, J. L., Riley, P., and Griffee, J. W.: Solar Wind Electron Proton Alpha Monitor (SWEPAM) for the Advanced Composition Explorer, Space Sci. Rev., 86, 563-612, 1998.

McFadden, J. P., Carlson, C. W., Larson, D., Ludlam, M., Abiad, R., Elliott, B., Turin, P., Marckwordt, M., and Angelopoulos, V.: The THEMIS ESA Plasma Instrument and In-flight Calibration, Space Sci. Rev., 141, 277-302, 2008.

Němeček, Z., Šafránková, J., Zastenker, G. N., Pišoft, P., Paularena, K. I., and Richardson, J. D.: Observations of the radial magnetosheath profile and a comparison with gasdynamic model predictions, Geophys. Res. Lett., 27, 2801-2804, 2000.

Paschmann, G., Sckopke, N., Asbridge, J. R., Bame, S. J., and Gosling, J. T.: Energization of solar wind ions by reflection from the earth's bow shock, J. Geophys. Res., 85, 4689-4693, 1980.

Roelof, E. C. and Sibeck, D. G.: Magnetopause shape as a bivariate function of interplanetary magnetic field $B_{\mathrm{Z}}$ and solar wind dynamic pressure, J. Geophys. Res., 98, 21421-21450, 1993.

Savoini, P. and Lembege, B.: Electron dynamics in two- and onedimensional oblique supercritical collisionless magnetosonic shocks, J. Geophys. Res., 99, 6609-6635, 1994.

Schwartz, S. J.: Shock and Discontinuity Normals, Mach Numbers, and Related Parameters, Analysis Methods for Multi-Spacecraft Data, edited by: Paschmann, G. and Daly, P., ISSI Scientific Reports Series, ESA/ISSI, Vol. 1., ISBN 1608-280X, 1998, p. 249270, 1, 249-270, 1998.

Schwartz, S. J., Thomsen, M. F., Bame, S. J., and Stansberry, J.: Electron heating and the potential jump across fast mode shocks, J. Geophys. Res., 93, 12923-12931, 1988.

Scudder, J. D., Aggson, T. L., Mangeney, A., Lacombe, C., and Harvey, C. C.: The resolved layer of a collisionless, high beta, supercritical, quasi-perpendicular shock wave. I - RankineHugoniot geometry, currents, and stationarity, J. Geophys. Res., 91, 11019-11052, 1986a.

Scudder, J. D., Aggson, T. L., Mangeney, A., Lacombe, C., and Harvey, C. C.: The resolved layer of a collisionless, high beta, supercritical, quasi-perpendicular shock wave. II - Dissipative fluid electrodynamics, J. Geophys. Res., 91, 11053-11073, 1986 b.

Scudder, J. D., Mangeney, A., Lacombe, C., Harvey, C. C., and Wu, C. S.: The resolved layer of a collisionless, high beta, supercritical, quasi-perpendicular shock wave. III - Vlasov electrodynamics, J. Geophys. Res., 91, 11075-11097, 1986c.

Slavin, J. A. and Holzer, R. E.: Solar wind flow about the terrestrial planets. I - Modeling bow shock position and shape, J. Geophys. 
Res., 86, 11401-11418, 1981.

Smith, C. W., L'Heureux, J., Ness, N. F., Acuña, M. H., Burlaga, L. F., and Scheifele, J.: The ACE Magnetic Fields Experiment, Space Sci. Rev., 86, 613-632, 1998.
Wu, C. S., Winske, D., Tanaka, M., Papadopoulos, K., Akimoto, K., Goodrich, C. C., Zhou, Y. M., Tsai, S. T., Rodriguez, P., and Lin, C. S.: Microinstabilities associated with a high Mach number, perpendicular bow shock, Space Sci. Rev., 37, 63-109, 1984. 